\title{
Finite Self-Information
}

\author{
Denis R. Hirschfeldt \\ Department of Mathematics \\ University of Chicago, USA \\ drh@math.uchicago.edu \\ Rebecca Weber ${ }^{*}$ \\ Department of Mathematics \\ Dartmouth College, USA \\ rweber@math. dartmouth. edu
}

\begin{abstract}
We present a definition, due to Levin, of mutual information $I(A: B)$ for infinite sequences. We say that a set $A$ has finite self-information if $I(A: A)<\infty$. It is easy to see that every $K$-trivial set has finite self-information. We answer a question of Levin by showing that the converse does not hold. Finally, we investigate the connections between having finite self-information and other notions of weakness such as jump-traceability. In particular, we show that our proof can be adapted to produce a set that is low for both effective Hausdorff dimension and effective packing dimension, but not $K$-trivial.
\end{abstract}

Keywords: mutual information, $K$-triviality, Kolmogorov complexity, effective dimension, jump traceability

We think of the information content of a finite binary string $\sigma$ as its prefix-free Kolmogorov complexity $K(\sigma)$. The idea of using plain Kolmogorov complexity to measure information content was proposed by Kolmogorov in [10]; for both technical and philosophical reasons plain complexity is often replaced by prefix-free Kolmogorov complexity (as defined by Levin [12] and later Chaitin [3]) in this and many other applications. The mutual information of two finite strings $\sigma, \tau$ is $K(\sigma)+K(\tau)-K(\sigma, \tau)$, where $K(\sigma, \tau):=K(\langle\sigma, \tau\rangle)$ for a standard pairing function $\langle\cdot, \cdot\rangle$. (For more on Kolmogorov complexity and related concepts, see [15].)

The proper extension of this definition from the finite to the infinite case is not clear-cut. A computable infinite binary sequence should contain finitely-much information, as coded by its generating program. One of the most important notions in the theory of algorithmic randomness is that of a $K$-trivial sequence, which is an infinite sequence $A$ such that $K(A\lceil n) \leqslant K(n)+c$ for some constant $c$ independent of $n$ (see Chapter 11 of [6] for more information on $K$-triviality). Since we are using prefix-free Kolmogorov complexity to measure information, a $K$-trivial sequence should also contain finitely-much information, since $K$ cannot differentiate between such a sequence and a computable sequence. Conversely, any non- $K$-trivial sequence should possess infinite information. Our purpose in this paper is not to propose a definition for the information content of an individual sequence, though both $\sup \left\{K(\sigma)-K^{A}(\sigma): \sigma \in 2^{<\omega}\right\}$ and $\lim \sup _{n}(K(A\lceil n)-K(n))$ satisfy the conditions above, but these intuitions provide a means to judge the quality of a definition of mutual information for infinite sequences. (Here $K^{A}$ is prefix-free Kolmogorov complexity relativized to $A$.)

Several such definitions have been proposed by Levin [12-14]. In these papers, Levin has also argued for the importance of the notion of mutual information in a wide range of applications. We focus on the definition given by Levin in [12], and used in that paper to prove theorems on information conservation. This definition has a number of desirable properties, and in many ways fulfills the intuition for the infinite case. The mutual information of $A$ and $B$, denoted by $I(A: B)$ and defined in the following section, is symmetric up to a constant, and when $A$ and $B$ are

\footnotetext{
* Both authors were partially supported by a National Science Foundation Focused Research Group grant, DMS-0652521 and DMS-0653226. Hirschfeldt was also supported by NSF grants DMS-0801033 and DMS-1101458. Both authors also acknowledge the support of the American Institute of Mathematics, as the question answered in this paper was formulated during a meeting they attended at the AIM Research Conference Center.
} 
finite strings it simplifies to $K(A)+K(B)-K(A, B)$. Furthermore, if $A$ is $K$-trivial then $I(A: B)$ is finite for all $B$, and as proved below, if $A$ is not $K$-trivial then there is some $B$ for which $I(A: B)$ is infinite.

During the AIM Workshop in Effective Randomness (held at ARCC, Palo Alto, CA, 7-11 August 2006), which the authors attended, Levin asked whether $I(A: A)<\infty$ implies $A$ is $K$-trivial. Here we answer this question negatively, constructing a non- $K$-trivial set with finite self-information. Separating these classes leads us to explore, in the final section of the paper, the relationships between having finite self-information and other weakness notions. In particular, we show that our construction can be modified to produce non- $K$-trivial sets that are low for effective dimension, as defined in that section.

At the workshop Levin also asked the "soft" question of which definition of mutual information is the "right" one. Our result might be seen as evidence against the definition presented below. However, we may argue for its plausibility in the face of our result by considering the possession of encoded information. If the second author has a cookbook in Portuguese, full of recipes unfamiliar to her, this does not help her make any new dishes. However, she possesses information, as evidenced by the fact that teaming up with the first author-who knows Portuguese and hence can decode the recipes-she can make meals she could not previously. This information simply does not contribute to her self-information. This idea of "hidden information", which a sequence may not be powerful enough to extract from itself, but which may be extracted by another sequence of different computational power, manifests itself in the contrast between Proposition 1.3 and Theorem 2.2 below.

We will use some basic facts about Kolmogorov complexity and algorithmic randomness. As usual, we identify a set $A$ with the infinite sequence corresponding to its characteristic function. All our logarithms are taken with base 2. Terms and notation not defined below may be found in Downey and Hirschfeldt [6]. In particular, we will use the KC Theorem (Theorem 3.6.1 in [6]): The weight of a sequence of pairs $\left\langle n_{i}, \sigma_{i}\right\rangle_{i \in \omega}$, where the $n_{i}$ are natural numbers and the $\sigma_{i}$ are either finite binary strings or natural numbers, is $\sum_{i} 2^{-n_{i}}$. Recall that such a sequence is a $K C$ set if it is a computable enumeration and its weight is finite. The KC Theorem states that for such a set, $K\left(\sigma_{i}\right) \leqslant{ }^{+} n_{i}$ for all $i$. (Here and below, a superscript + on a relation means the relation holds up to an additive constant.) We will also use the Recursion Theorem in conjunction with the KC Theorem; for a discussion of this technique, see Section 3.6 in [6]. Another important tool will be the result due to Nies [19] that if $A$ is $K$-trivial then it is low for $K$; that is, $K^{A}(\sigma)={ }^{+} K(\sigma)$ for all $\sigma$.

\section{Mutual Information}

Definition 1.1 (Levin [12]). The mutual information of infinite binary sequences $A$ and $B$ is

$$
I(A: B)=\log \sum_{\sigma, \tau \in 2<\omega} 2^{-K(\sigma, \tau)-K^{A}(\sigma)-K^{B}(\tau)+K(\sigma)+K(\tau)} .
$$

Note that this definition is independent of the choice of universal prefix-free machine up to an additive constant. Levin [12] showed that if we replace $A$ and $B$ in the above definition by finite strings $\alpha$ and $\beta$, then $I(\alpha: \beta)$ is equal (up to an additive constant) to the usual definition of mutual information for finite strings, namely $K(\alpha)+K(\beta)-$ $K(\alpha, \beta)$.

We may think of this definition as follows. One way to measure the mutual information of individuals $A$ and $B$ is to run through all pairs of subjects, and for each pair $(\sigma, \tau)$, to determine how much information $A$ and $B$ have about $\sigma$ and $\tau$, respectively. The weight we should give to each pair depends on the relationship between its elements; the more closely related $\sigma$ and $\tau$ are, the more the pair should matter in our computation. For example, the fact that $A$ knows about snakes and $B$ knows about lizards should contribute more to the computation of the mutual information of $A$ and $B$ than the fact that $A$ knows about snakes and $B$ knows about doorknobs. The degree of relatedness of strings $\sigma$ and $\tau$ is represented by their mutual information $I(\sigma: \tau)=K(\sigma)+K(\tau)-K(\sigma, \tau)$; in the extreme case $\sigma=\tau$ this is essentially $K(\sigma)$, the total information in $\sigma$, and if $\sigma$ and $\tau$ are mutually relatively random it is essentially 0 . From this quantity we want to include only the portion corresponding to $A$ 's knowledge of $\sigma$ and $B$ 's knowledge of $\tau$. Since the relativized complexity $K^{A}(\sigma)$ may be thought of as measuring what $A$ does 
not know about $\sigma$, we subtract it from $I(\sigma: \tau)$, and similarly we subtract $K^{B}(\tau)$. See [12] for further comments on the motivations behind this definition, and a way to think of it in terms of a priori probabilities.

The next two facts (the first of which was noted by several people at the aforementioned AIM workshop) show that this definition gives, at least to some degree, the desired split between finite information and infinite information.

Proposition 1.2. If $A$ is $K$-trivial then $I(A: B)$ is finite for all $B$.

Proof. Let $A$ be $K$-trivial and $B$ be any set. As mentioned in the introduction, $K^{A}(\sigma)={ }^{+} K(\sigma)$ for all $\sigma \in 2^{<\omega}$. Since $K(\sigma, \tau) \geqslant^{+} K(\tau)$ for all $\sigma$ and $\tau$, we see that $I(A: B)$ is bounded above (up to a constant) by

$$
\log \sum_{\tau \in 2<\omega} 2^{-K^{B}(\tau)}
$$

which is finite by Kraft's Inequality (see Section 3.6 of [6]).

Proposition 1.3 (with Jan Reimann). If $A$ is not $K$-trivial, there exists $B$ such that $I(A: B)=\infty$. In particular, any $B \geqslant_{T} A \oplus \emptyset^{\prime}$ has infinite mutual information with $A$.

Proof. Let $B \geqslant_{\mathrm{T}} A \oplus \emptyset^{\prime}$; note that $B$ can compute $K$ and approximate $K^{A}$ from above. We choose a subsequence of initial segments of $A$ and use $B$ to compress them highly, making the mutual information sum diverge. To do so, we build a KC set $M$ relative to $B$.

Since $A$ is not $K$-trivial, for each $n$ we have some $\tau_{n} \prec A$ such that $K\left(\tau_{n}\right)-K^{A}\left(\tau_{n}\right) \geqslant n$. Since $B$ can compute $K$, and its approximation to $K^{A}(\tau)$ for any $\tau$ can only decrease, $B$ can effectively find such a $\tau_{n}$ for each $n$. Let $M=\left\{\left\langle n, \tau_{n}\right\rangle \mid n \in \omega\right\}$. Then $M$ is a KC set relative to $B$, and hence $K^{B}\left(\tau_{n}\right) \leqslant^{+} n$ for all $n$. Since $K\left(\tau_{n}, \tau_{n}\right)={ }^{+} K\left(\tau_{n}\right)$, we have

$$
I(A: B) \geqslant \log \sum_{n} 2^{K\left(\tau_{n}\right)-K^{A}\left(\tau_{n}\right)-K^{B}\left(\tau_{n}\right)-O(1)} \geqslant \log \sum_{n} 2^{n-n-O(1)}=\infty .
$$

It is open whether there is an $A$ for which $B \geqslant_{\mathrm{T}} A \oplus \emptyset^{\prime}$ is actually required to obtain $I(A: B)=\infty$. We show in the following section that $B=A$ does not necessarily suffice, though, as noted by Levin [12], every 1-random set has infinite mutual information with itself and hence the cases for which $B=A$ does not suffice have measure zero.

One may ask whether letting the sum in Definition 1.1 range over all pairs $\sigma, \tau \in 2^{<\omega}$ is necessary. We may simplify to only $\sigma=\tau$, obtaining the following definition.

Definition 1.4 (Levin [12]). The simplified mutual information of infinite binary sequences $A$ and $B$ is

$$
I^{\prime}(A: B)=\log \sum_{\sigma \in 2<\omega} 2^{-K^{A}(\sigma)-K^{B}(\sigma)+K(\sigma)} .
$$

It is clear that $I^{\prime}(A: B)=\infty$ implies $I(A: B)=\infty$. However, it is open whether the converse implication holds, both in general and for the special case of $A=B$, where the answer may differ. (Proposition 1.3 does hold for $I^{\prime}$ in place of $I$, with essentially the same proof.) A priori, what $I(A: B)$ accounts for that $I^{\prime}(A: B)$ does not, at least not obviously, is information about related subjects. If $A$ knows about snakes and $B$ about lizards, these are sufficiently related topics that $A$ and $B$ share significant information. However, there does exist a single "snakes and lizards" string about which they should both know, and hence the plausibility of the idea that $I^{\prime}(A: B)=\infty \Longleftrightarrow$ $I(A: B)=\infty$.

A related question also comes from [12]. As mentioned above, for finite strings $\sigma, \tau$, we have $I(\sigma: \tau)=^{+}$ $K(\sigma)+K(\tau)-K(\sigma, \tau)$. The corresponding equality for $I^{\prime}(\sigma: \tau)$ appears to be open. 


\section{Finite Self-Information}

As mentioned above, $I(A: A)<\infty$ for all $K$-trivial $A$, and $I(A: A)=\infty$ for all 1-random $A$. We make the following definition.

Definition 2.1. A set $A$ has finite self-information if $I(A: A)<\infty$.

Our main result, which we prove in this section, shows that this class of sets properly contains the $K$-trivials, answering the aforementioned question of Levin.

Theorem 2.2. There is a non-K-trivial set A that has finite self-information.

Relationships between the class of sets that have finite self-information and other classes defined by weakness notions are explored in the next section. The structure of this class is largely unknown, and the mutual information of sets that have finite self-information with sets other than themselves may be of interest. For example, perhaps a set $A$ that has finite self-information but is not $K$-trivial requires $B \geqslant_{\mathrm{T}} A \oplus \emptyset^{\prime}$ to have $I(A: B)=\infty$. Less drastically, perhaps if both $A$ and $B$ have finite self-information then $I(A: B)<\infty$ always holds.

We need a particular function for the proof of Theorem 2.2.

Lemma 2.3. There is a function $f$ such that $\sum_{\sigma, \tau} 2^{-K(\sigma, \tau)+f(\sigma)+f(\tau)}$ converges and $f$ has a computable approximation for which

$$
(\forall i)\left(\forall^{\infty} \sigma\right)(\forall s)\left[f_{s}(\sigma)>i\right] .
$$

Before proving this lemma, we note its connection with finite self-information.

Lemma 2.4. If $\sum_{\sigma, \tau} 2^{-K(\sigma, \tau)+f(\sigma)+f(\tau)}$ converges and

$$
K(\sigma) \leqslant K^{A}(\sigma)+f(\sigma)
$$

for all $\sigma \in 2^{<\omega}$, then $A$ has finite self-information.

Proof. For such an $A$, we have

$$
\begin{aligned}
I(A: A) & =\log \sum_{\sigma, \tau} 2^{-K(\sigma, \tau)-K^{A}(\sigma)-K^{A}(\tau)+K(\sigma)+K(\tau)} \\
& \leqslant \log \sum_{\sigma, \tau} 2^{-K(\sigma, \tau)+f(\sigma)+f(\tau)+O(1)}<\infty .
\end{aligned}
$$

To prove Lemma 2.3, we use two auxiliary functions, given by the following lemmas.

Lemma 2.5. There is a function $h$ such that $\sum_{\sigma} 2^{-K(\sigma)+2 h(\sigma)}$ converges and $h$ has a computable approximation for which

$$
(\forall i)\left(\forall^{\infty} \sigma\right)(\forall s)\left[h_{s}(\sigma)>i\right]
$$

Note that if we were working with the simplified mutual information of Definition 1.4, Lemma 2.4 would hold with this function $h$ in place of $f$.

Proof of Lemma 2.5. Noneffectively, $h$ is defined as follows. Fix an approximation $\Omega_{s}$ to $\Omega$. (Here $\Omega$ is the halting probability of a universal prefix-free machine; see [6] for more on this number.) We assume this approximation is given by a universal prefix-free machine $U$ subject to the usual convention that if $U_{s}(\tau) \downarrow=\sigma$, then $s>|\sigma|$. Let $s_{0}, s_{1}, \ldots$ be the nondecreasing sequence such that $s_{0}=0$ and $s_{i+1}$ is the first stage at which our approximation is 
within $8^{-i}$ of $\Omega$. Given $\sigma$, let $t$ be the first stage at which $K_{t}(\sigma)=K(\sigma)$. For the unique $i$ such that $s_{i}<t \leqslant s_{i+1}$, let $h(\sigma)=i$.

If $h(\sigma)=i$, then there is a $\tau$ such that $|\tau|=K(\sigma)$ and $U(\tau) \downarrow=\sigma$ but $U_{s_{i}}(\tau) \uparrow$. Thus, $\sum_{\sigma: h(\sigma)=i} 2^{-K(\sigma)} \leqslant \Omega-$ $\Omega_{s_{i}}$. If $i>0$, then this quantity is at most $8^{-i}$, so

$$
\begin{aligned}
\sum_{\sigma} 2^{-K(\sigma)+2 h(\sigma)} & \leqslant \sum_{\sigma: h(\sigma)=0} 2^{-K(\sigma)}+\sum_{i>0} \sum_{\sigma: h(\sigma)=i} 2^{2 i} 2^{-K(\sigma)} \\
& \leqslant \Omega+\sum_{i>0} 4^{i} 8^{-i}=\Omega+\sum_{i>0} 2^{-i}<\infty .
\end{aligned}
$$

To approximate $h$, we first approximate the sequence $\left(s_{i}\right)$. Let $s_{0}[t]=0$ for all $t$. For $i>0$ and $t$, let $s_{i}[t]$ be the least $s$ such that $\Omega_{t}-\Omega_{s} \leqslant 8^{-i}$. Clearly, $s_{i}[t] \leqslant s_{i}[t+1]$ for all $i$ and $t$, and $\lim _{t} s_{i}[t]=s_{i}$ for all $i$.

To define $h_{t}(\sigma)$, let $t^{\prime} \leqslant t$ be the most recent $t^{\prime}>|\sigma|$ at which $K_{t^{\prime}}(\sigma)<K_{t^{\prime}-1}(\sigma)$, or $t^{\prime}=|\sigma|+1$ if there is none. Let $i$ be such that $s_{i}[t]<t^{\prime} \leqslant s_{i+1}[t]$, and set $h_{t}(\sigma)=i$.

It is clear that this approximation is computable and has limit $h$. If $|\sigma| \geqslant s_{i}$ then $|\sigma| \geqslant s_{i}[t]$ for all $t$, so $h_{t}(\sigma) \geqslant i$ for all $t$. Thus, our approximation to $h$ satisfies (3).

Lemma 2.6. There are a 2-place function $g$ and a constant $r$ such that for every $\sigma$ we have $\sum_{\tau} 2^{-K(\sigma, \tau)+g(\sigma, \tau)}<$ $2^{-K(\sigma)+r}$, and furthermore $g$ has a computable approximation such that for each $\sigma$,

$$
(\forall i)\left(\forall^{\infty} \tau\right)(\forall s)\left[g_{s}(\sigma, \tau)>i\right]
$$

Proof. The proof of this lemma is similar to that of Lemma 2.5. Indeed, it would not be difficult to obtain Lemma 2.5 as a corollary to this lemma, but we chose not do so for clarity of exposition.

Let $\Omega^{\sigma}$ be the sum of $2^{-|\rho|}$ over all $\rho$ that are descriptions of $(\sigma, \tau)$ for some $\tau$. The function $\sigma \mapsto-\log \Omega^{\sigma}$ is a complexity measure, so by the minimality of $K$ among such measures, there is a constant $r$ such that $2^{-K(\sigma)+r}>\Omega^{\sigma}$ for all $\sigma$. (Here $\log r$ means the base $2 \log$ arithm of $r$, rounded up to the nearest larger integer. See Section 3.7 of [6] for more details on the minimality of $K$ among complexity measures.) We can choose $r$ so that $2^{-K(\sigma)+r}-\Omega^{\sigma}>1$.

Noneffectively, $g$ is defined as follows. Fix uniform approximations $\Omega_{s}^{\sigma}$ to $\Omega^{\sigma}$ for each $\sigma$, with the same assumption as in the proof of Lemma 2.5. For a given $\sigma$, let $s_{0}, s_{1}, \ldots$ be the nondecreasing sequence such that $s_{0}=0$ and $s_{i+1}$ is the first stage at which our approximation is within $4^{-i}$ of $\Omega^{\sigma}$. Given $\sigma$, let $t$ be the first stage at which $K_{t}(\sigma, \tau)=K(\sigma, \tau)$. For the unique $i$ such that $s_{i}<t \leqslant s_{i+1}$, let $g(\sigma, \tau)=i$.

If $g(\sigma, \tau)=i$, then there is a $\rho$ such that $|\rho|=K(\sigma, \tau)$ and $U(\rho) \downarrow=(\sigma, \tau)$ but $U_{s_{i}}(\rho) \uparrow$, where $U$ is our fixed universal prefix-free machine. Thus, for each $\sigma$, we have $\sum_{\tau: g(\sigma, \tau)=i} 2^{-K(\sigma, \tau)} \leqslant \Omega^{\sigma}-\Omega_{s_{i}}^{\sigma}$. If $i>0$, then this quantity is at most $4^{-i}$, so

$$
\begin{aligned}
\sum_{\tau} 2^{-K(\sigma, \tau)+g(\sigma, \tau)} & \leqslant \sum_{\tau: h(\sigma, \tau)=0} 2^{-K(\sigma, \tau)}+\sum_{i>0} \sum_{\tau: g(\sigma, \tau)=i} 2^{i} 2^{-K(\sigma, \tau)} \\
& \leqslant \Omega^{\sigma}+\sum_{i>0} 2^{i} 4^{-i}=\Omega^{\sigma}+\sum_{i>0} 2^{-i}=\Omega^{\sigma}+1<2^{-K(\sigma)+r} .
\end{aligned}
$$

To approximate $g$, given $\sigma$, we first approximate the sequence $\left(s_{i}\right)$. Let $s_{0}[t]=0$ for all $t$. For $i>0$ and $t$, let $s_{i}[t]$ be the least $s$ such that $\Omega_{t}^{\sigma}-\Omega_{s}^{\sigma} \leqslant 4^{-i}$. Clearly, $s_{i}[t] \leqslant s_{i}[t+1]$ for all $i$ and $t$, and $\lim _{t} s_{i}[t]=s_{i}$ for all $i$.

To define $g_{t}(\sigma, \tau)$, let $t^{\prime} \leqslant t$ be the most recent $t^{\prime}>|\tau|$ at which $K_{t^{\prime}}(\sigma, \tau)<K_{t^{\prime}-1}(\sigma, \tau)$, or $t^{\prime}=|\tau|+1$ if there is none. Let $i$ be such that $s_{i}[t]<t^{\prime} \leqslant s_{i+1}[t]$, and set $g_{t}(\sigma, \tau)=i$.

It is clear that this approximation is computable and has limit $g$. For a fixed $\sigma$ and $s_{i}$ as above, if $|\tau| \geqslant s_{i}$ then $|\tau| \geqslant s_{i}[t]$ for all $t$, so $g_{t}(\sigma, \tau) \geqslant i$ for all $t$. Thus, our approximation to $g$ satisfies (4). 
Proof of Lemma 2.3. Let $h$ be as in Lemma 2.5 and $g$ as in Lemma 2.6. We may assume that $g(\sigma, \sigma)=0$ for all $\sigma$. Let $<_{l}$ be the length-lexicographic ordering on strings. Define

$$
f(\tau)=\min _{\sigma \leqslant l \tau}(h(\sigma)+g(\sigma, \tau))
$$

Letting $r$ be as in Lemma 2.6, we have

$$
\begin{aligned}
\sum_{\sigma, \tau} 2^{-K(\sigma, \tau)+f(\sigma)+f(\tau)} & \leqslant 2 \sum_{\sigma} \sum_{\tau \geqslant l \sigma} 2^{-K(\sigma, \tau)+f(\sigma)+f(\tau)} \\
& =2 \sum_{\sigma} 2^{f(\sigma)} \sum_{\tau \geqslant l \sigma} 2^{-K(\sigma, \tau)+f(\tau)} \leqslant 2 \sum_{\sigma} 2^{h(\sigma)} \sum_{\tau \geqslant l \sigma} 2^{-K(\sigma, \tau)+h(\sigma)+g(\sigma, \tau)} \\
& \leqslant 2 \sum_{\sigma} 2^{2 h(\sigma)} 2^{-K(\sigma)+r}=2^{r+1} \sum_{\sigma} 2^{-K(\sigma)+2 h(\sigma)}
\end{aligned}
$$

which converges by the choice of $h$.

Let $f_{s}(\tau)=\min _{\sigma \leqslant l}\left(h_{s}(\sigma)+g_{s}(\sigma, \tau)\right)$, where $h_{s}$ and $g_{s}$ satisfy (3) and (4), respectively. For a given $i$, there are only finitely many $\sigma$ such that $h_{s}(\sigma)$ is ever less than $i$. For each such $\sigma$, there are only finitely many $\tau$ such that $g_{s}(\sigma, \tau)$ is ever less than $i$. Thus, for all but finitely many $\tau$, we have $h_{s}(\sigma)+g_{s}(\sigma, \tau) \geqslant i$ for all $\sigma$ and $s$. Hence, $f_{s}$ has property (1).

We assume without loss of generality that the function $f$ from Lemma 2.3 is approximated from above; that is, that $f_{s+1}(\sigma) \leqslant f_{s}(\sigma)$ for all $s$ and $\sigma$. If it is not originally, then we may replace $f(\sigma)$ with $\min _{t} f_{t}(\sigma)$ and $f_{s}(\sigma)$ with $\min _{t \leqslant s} f_{t}(\sigma)$, and the desired properties will still hold.

Before proceeding to the proof of Theorem 2.2, we establish a couple of useful lemmas.

Lemma 2.7. By sufficiently speeding up our enumeration of stages, we may assume that there is a $b$ such that for each $n, c$, and $v$, the number of strings $\tau$ of length $n$ for which there is an s with $K_{s}(\tau) \leqslant K_{s}(n)+c=v$ is bounded above by $2^{c+b}$.

Proof. By Chaitin's Counting Lemma (Theorem 3.7 .6 in [6]), there exists a constant $b$ such that for every $n$ there are fewer than $2^{c+b}$-many $\tau$ of length $n$ with $K(\tau) \leqslant K(n)+c$, so we may assume the approximation to $K$ is sped up so that for each $s$ we have fewer than $2^{c+b}$-many $\tau$ of length $n$ with $K_{s}(\tau) \leqslant K_{s}(n)+c$. If $t>s$ and $K_{t}(n)=K_{s}(n)$, then $K_{s}(\tau) \leqslant K_{s}(n)+c$ implies that $K_{t}(\tau) \leqslant K_{t}(n)+c$, so for each $v$, there are fewer than $2^{c+b}$-many $\tau$ of length $n$ for which there is an $s$ with $K_{s}(\tau) \leqslant K_{s}(n)+c=v$.

Define an oracle $K C$ set to be a computable enumeration of triples $\left\langle n_{i}, \sigma_{i}, \tau_{i}\right\rangle$ such that for the finitely-many triples listed at stage $s$ (if any), we have $\left|\tau_{i}\right|=s$, and there is a $p$ such that for any $X \in 2^{\omega}$, we have $\sum_{\tau_{i} \prec X} 2^{-n_{i}} \leqslant p$. The proof of the following result is a straightforward modification of the proof of the KC Theorem, as in Section 3.6 of [6].

Lemma 2.8. We can speed up our enumeration of stages sufficiently to ensure that for each oracle KC set $\left\langle n_{i}, \sigma_{i}, \tau_{i}\right\rangle_{i \in \omega}$, there is a $t$ such that if $\left\langle n_{i}, \sigma_{i}, \tau_{i}\right\rangle$ is listed at stage $s>t$ then $K_{s+1}^{\tau_{i}}\left(\sigma_{i}\right) \leqslant{ }^{+} n_{i}$.

We are now ready to prove our main result.

Proof of Theorem 2.2. We assume in our construction that we have sped up our enumeration of stages so that Lemmas 2.7 and 2.8 apply. We also assume the usual convention that the use of any computation at stage $s$ is at most $s$. By $A_{s}$ we mean what we have enumerated into $A$ by the beginning of stage $s$. For the largest number $r$ seen in the construction by the beginning of stage $s$, we identify $A_{s}$ with the finite string $A_{s}\lceil r+1$. 
To make $A$ have finite self-information, we use Lemma 2.4. We ensure (2) in the usual way, building a KC set $M$ by enumerating axioms $\left\langle K_{s}^{A_{s}}(\sigma)+f_{s}(\sigma), \sigma\right\rangle$ whenever needed.

To make $A$ non- $K$-trivial, for each constant $c$ we define some $n_{c}$ such that either $K\left(A\left\lceil n_{c}\right)>K\left(n_{c}\right)+c\right.$ or $K^{A}\left(n_{c}\right)<K\left(n_{c}\right)-c$. (If the first disjunct holds for infinitely-many $c$, then $A$ is not $K$-trivial; if the second one holds for infinitely-many $c$, then $A$ is not low for $K$, and hence is again not $K$-trivial.)

For each $c$, we have a strategy for defining such an $n_{c}$. This will be a finite injury priority construction; as usual, the $c$ strategy has priority over the $\hat{c}$ strategy if $c<\hat{c}$. The module for a single $c$ is as follows. Let $C(x, s)$ be the set of all strings $\sigma$ such that there exists a minimal-length $A_{s}$-description of $\sigma$ at stage $s$ with use $>x$. These are the strings $\sigma$ for which the approximation to $K^{A}(\sigma)$ might go up after stage $s$ if we put $x$ into $A$ after that stage.

We will enumerate numbers $m_{c, i}$ and $n_{c, i}$ for $i$ in some initial segment of $\omega$. By the KC Theorem, there is a $d$ (independent of $c$ ) such that $K\left(n_{c, i}\right) \leqslant c+i+d$ for all $i$ for which $n_{c, i}$ is defined. By the Recursion Theorem, we may assume that we know $d$. We may also assume that we have sped our enumeration of stages sufficiently so that $K_{s}\left(n_{c, i}\right) \leqslant c+i+d$ for all $s$ such that $n_{c, i}$ is defined.

Begin with $i=0$. Let $b$ be as in Lemma 2.7. Pick fresh, large numbers $m_{c, i}, n_{c, i}$ such that the interval $\left(m_{c, i}, n_{c, i}\right)$ contains $(c+i+d) 2^{c+b}$-many numbers. (As we will show below, the size of this interval is an upper bound on how often we may want to change $A$ to ensure that $K_{s}\left(A_{s}\left\lceil n_{c, i}\right)>K_{s}\left(n_{c, i}\right)+c\right.$. There will be at most $2^{c+b}$ many changes needed for each new value of $K_{s}\left(n_{c, i}\right)$.)

There are three actions we might take on behalf of $c$. The first two ensure that either $A$ is not low for $K$ with constant $c$ or $A$ is not $K$-trivial with constant $c$. The third will be needed in showing that $M$ is indeed a KC set. It ensures that if we enumerate a number into $A$ for the sake of $c$, and this enumeration causes $\left\langle K_{s}^{A_{s}}(\sigma)+f_{s}(\sigma), \sigma\right\rangle$ to enter $M$, then $f_{s}(\sigma)$ is not too small. (As we will show below, requiring that $f_{s}(\sigma) \geqslant 4 c$ suffices.)

First, whenever we see

$$
K_{s+1}^{A_{s}}\left(n_{c, i}\right)<K_{s+1}\left(n_{c, i}\right)-c,
$$

we suspend the action of the $c$ strategy and restrain $A$ up to the use of the computation witnessing the current value of $K_{s+1}^{A_{s}}\left(n_{c, i}\right)$ by initializing all weaker-priority requirements. (The reason we use $K_{s+1}$ here instead of $K_{s}$ will be clear when we apply Lemma 2.8 below.) The suspension continues until, if ever, (5) no longer holds. If the strategy remains suspended forever, then $n_{c, i}$ witnesses that $A$ is not low for $K$ with constant $c$.

Second, if action on behalf of $c$ is not suspended, whenever we see $K_{s}\left(A_{s}\left\lceil n_{c, i}\right) \leqslant K_{s}\left(n_{c, i}\right)+c\right.$ we enumerate a new number from $\left(m_{c, i}, n_{c, i}\right)$ into $A$. We will show that we never run out of space for these enumerations, and hence this action ensures that if the $c$ strategy is not eventually suspended forever, then $K\left(A\left\lceil n_{c, i}\right)>K\left(n_{c, i}\right)+c\right.$, and hence $A$ is not $K$-trivial with constant $c$.

Third, if we ever see $f_{s}(\sigma)<4 c$ for any $\sigma \in C\left(m_{c, i}, s\right)$, we initialize the $c$ strategy by increasing $i$ by one and picking fresh, large $m_{c, i}, n_{c, i}$ such that again the interval $\left(m_{c, i}, n_{c, i}\right)$ contains $(c+i+d) 2^{c+b}$-many numbers. We also initialize weaker-priority strategies, which restrains $A\left\lceil m_{c, i}\right.$ for the new value of $m_{c, i}$, to attempt to ensure that the $\sigma \in C\left(m_{c, i}, s\right)$ have minimal-length $A$-descriptions with use $>m_{c, i}$. This action ensures that we eventually find an $i$ such that $f(\sigma) \geqslant 4 c$ for all $\sigma \in C\left(m_{c, i}, s\right)$, since it ensures that each $\sigma$ can be in only finitely-many $C\left(m_{c, i}, s\right)$.

We now proceed with the full construction and its verification.

Construction. Let $b$ and $d$ be as above. Each strategy has a counter (corresponding to the number $i$ above), which is initially set to 0 . Initially no $m_{c, i}$ or $n_{c, i}$ is defined, and $A$ and $M$ are both empty. At stage $s$, act as follows.

First, for any $\sigma$ such that $K_{s}^{A_{s}}(\sigma)+f_{s}(\sigma)<K_{t}^{A_{t}}(\sigma)+f_{t}(\sigma)$ for all $t<s$ (where we take $K_{t}^{A_{t}}(\sigma)+f_{t}(\sigma)=\infty$ if $t \leqslant|\sigma|)$, enumerate $\left\langle K_{s}^{A_{s}}(\sigma)+f_{s}(\sigma), \sigma\right\rangle$ into $M$.

Next, proceed as follows for each $c$. Let $i$ be the current value of the counter associated with $c$. If the $c$ strategy was suspended at the end of stage $s-1$ but $K_{s+1}\left(n_{c, i}\right)<K_{s}\left(n_{c, i}\right)$, then declare the $c$ strategy not to be suspended. If the $c$ strategy is not currently suspended and $K_{s+1}^{A_{s}}\left(n_{c, i}\right)<K_{s+1}\left(n_{c, i}\right)-c$, then declare the $c$ strategy to be suspended. We say that this strategy is newly suspended at stage $s$. 
Finally, act for the least $c$ that requires attention, where the attention that may be required and the action to be taken are as follows. Here $i$ is the current value of the counter associated with $c$. When we say that we initialize a strategy, we mean that we increase its counter and declare it not to be suspended.

(i) The numbers $m_{c, i}, n_{c, i}$ are not defined. In this case, define $m_{c, i}$ and $n_{c, i}$ to be fresh, large numbers such that the interval $\left(m_{c, i}, n_{c, i}\right)$ contains $(c+i+d) 2^{c+b}$-many numbers.

(ii) The $c$ strategy is newly suspended at $s$. In this case, initialize all weaker-priority strategies.

(iii) The $c$ strategy is not suspended, the numbers $m_{c, i}, n_{c, i}$ are defined, and there is a $\sigma \in C\left(m_{c, i}, s\right)$ with $f_{s}(\sigma)<$ $4 c$. In this case, initialize the $c$ strategy and all weaker-priority strategies.

(iv) The $c$ strategy is not suspended, the numbers $m_{c, i}, n_{c, i}$ are defined, and $K_{s}\left(A_{s}\left\lceil n_{c, i}\right) \leqslant K_{s}\left(n_{c, i}\right)+c\right.$. In this case, enumerate a new number from $\left(m_{c, i}, n_{c, i}\right)$ into $A$ (we will show below that there always is such a number not yet in $A$ ), and initialize all weaker-priority strategies.

Verification. The following lemma shows that $A$ is not $K$-trivial, as it implies that there are either infinitely many $c$ such that $A$ is not low for $K$ with constant $c$, or infinitely many $c$ such that $A$ is not $K$-trivial with constant $c$.

Lemma 2.9. For each $c$, the $c$ strategy acts only finitely often, and its counter has a final value $i$. Either the $c$ strategy is eventually suspended forever, in which case $K^{A}\left(n_{c, i}\right)<K\left(n_{c, i}\right)-c$, or it ensures that $K\left(A\left\lceil n_{c, i}\right)>K\left(n_{c, i}\right)+c\right.$.

Proof. Fix $c$ and suppose by induction that all stronger-priority strategies have ceased acting by some stage $s^{*}$. The $c$ strategy is initialized at a stage $s \geqslant s^{*}$ only when there is a $\sigma \in C\left(m_{c, i}, s\right)$ with $f_{s}(\sigma)<4 c$, where $i$ is the value of its counter at stage $s$. Let us say that such a $\sigma$ causes this initialization. It is easy to check from the construction that the numbers put in by strategies for $\hat{c} \geqslant c$ at stages greater than or equal to $s$ are all greater than $m_{c, i}$. Thus, by the assumption that stronger-priority strategies have stopped acting, $A$ cannot change below $m_{c, i}$ at any stage greater than or equal to $s$, so the minimal-length $A_{s}$-description witnessing the fact that $\sigma \in C\left(m_{c, i}, s\right)$ is in fact an $A$-description. It follows that each $\sigma$ can cause only finitely-many initializations of the $c$ strategy. By property (1) of $f$ from Lemma 2.3, there are only finitely-many $\sigma$ such that $f_{s}(\sigma)<4 c$ for some $s$; that is, there are only finitely-many $\sigma$ that can ever cause an initialization of the $c$ strategy. Thus the $c$ strategy is initialized only finitely often.

Let $s^{* *}$ be the last stage at which $c$ is initialized (or 0 if there is no such stage). Let $i$ be the value of the counter associated with $c$ at stage $s^{* *}$, and note that this is the final value of this counter. If the $c$ strategy is newly suspended at a stage $s>s^{* *}$, then $K_{s+1}^{A_{s}}\left(n_{c, i}\right)<K_{s+1}\left(n_{c, i}\right)-c$. Since weaker-priority strategies are initialized at stage $s$, as long as the $c$ strategy is suspended, $A$ will not change below the use of the computation that gives $K_{s+1}^{A_{s}}\left(n_{c, i}\right)$ its value. If the $c$ strategy is permanently suspended after stage $s$, then $K\left(n_{c, i}\right)=K_{s+1}\left(n_{c, i}\right)$, so $K^{A}\left(n_{c, i}\right) \leqslant K_{s+1}^{A_{s}}\left(n_{c, i}\right)<K\left(n_{c, i}\right)-c$. Otherwise, $K\left(n_{c, i}\right)<K_{s+1}\left(n_{c, i}\right)$, which can happen for only finitely-many $s$. Thus either the $c$ strategy is eventually suspended forever, in which case $K^{A}\left(n_{c, i}\right)<K\left(n_{c, i}\right)-c$ and the $c$ strategy acts only finitely often, or the $c$ strategy is suspended for only finitely-many stages.

So suppose we are in the latter case. Every time the $c$ strategy enumerates a number in $\left(m_{c, i}, n_{c, i}\right)$ into $A$ at stage $s$, it is because $K_{s}\left(A_{s} \uparrow n_{c, i}\right) \leqslant K_{s}\left(n_{c, i}\right)+c$. This enumeration ensures that $A_{t}\left\lceil n_{c, i} \neq A_{s}\left\lceil n_{c, i}\right.\right.$ for all $t>s$. By our choice of $b$ and the assumption that $K_{t}\left(n_{c, i}\right) \leqslant c+i+d$ for all $t$, there are no more than $(c+i+d) 2^{c+b}$-many $\tau$ of length $n_{c, i}$ with $K_{s}(\tau) \leqslant K_{s}\left(n_{c, i}\right)+c$ for some $s$, so there are no more than $(c+i+d) 2^{c+b}$-many such enumerations. Thus the $c$ strategy eventually stops acting, and for all sufficiently large $s$, we have $K_{s}\left(A_{s}\left\lceil n_{c, i}\right)>K_{s}\left(n_{c, i}\right)+c\right.$, whence $K\left(A\left\lceil n_{c, i}\right)>K\left(n_{c, i}\right)+c\right.$.

It remains to show that $A$ has finite self-information. We begin with an auxiliary lemma.

Lemma 2.10. Let $i_{s}^{c}$ be the value of the counter associated with $c$ at stage $s$. There are $k$ and $t$ such that for each $c$ and $s>t$ for which $m_{c, i_{s}}, n_{c, i_{s}^{c}}$ are defined, we have

$$
2^{-K_{s+1}^{A_{s}}\left(n_{c, i_{s}^{c}}\right)} \geqslant 2^{-c-k} \sum_{\sigma \in C\left(m_{c, i_{s}}, s\right)} 2^{-K_{s}^{A_{s}}(\sigma)} .
$$


Proof. We enumerate an oracle KC set $L$. By Lemma 2.8, there are $k$ and $t$ such that if $\left\langle n_{i}, \sigma_{i}, \tau_{i}\right\rangle$ enters $L$ at stage $s>t$ then $K_{s+1}^{\tau_{i}}\left(\sigma_{i}\right) \leqslant n_{i}+k$. At each stage $s$ and for each $c \leqslant s$, proceed as follows. For each $\sigma$ in $C\left(m_{c, i_{s}}, s\right)$, let $\mu$ be a witness to $\sigma$ being in that set; that is, a minimal length $A_{s}$-description of $\sigma$ at stage $s$ with use $>m_{c, i_{s}^{c}}$. Put $\mu$ into an auxiliary set $S_{c, s}$. Let $u_{c, s}$ be the measure of the open set in Cantor space determined by $S_{c, s}$; in other words, $u_{c, s}=\sum_{\sigma \in C\left(m_{c, i, s}, s\right)} 2^{-K_{s}^{A_{s}}(\sigma)}$. Let $j$ be least such that $\left\langle j, n_{c, i, c}, \tau\right\rangle$ is currently in $L$ for some $\tau \prec A_{s}$. If $c-\log u_{c, s}<j$, then put $\left\langle c-\log u_{c, s}, n_{c, i_{s}}, A_{s}\right\rangle$ into $L$. (Here $\log r$ means the base $2 \operatorname{logarithm}$ of $r$, rounded up to the nearest largest integer.)

If $s<u$ is such that $A_{s} \prec A_{u}$ and $\mu \in S_{c, s}$, then $\mu$ is still an $A_{u}$-description, since its use as an $A_{s}$-description has not changed between stages $s$ and $u$. Thus, if $\nu \in S_{c, u}$ and $\nu \neq \mu$, then $\mu$ and $\nu$ are incompatible. This argument shows that for any $X$, the set $\bigcup_{A_{s} \prec X} S_{c, s}$ is prefix-free. Thus the sum of $u_{c, s}$ taken over all $s$ such that $A_{s} \prec X$ is bounded by 1 . Hence the sum of $2^{-j}$ taken over all axioms in $L$ of the form $\left\langle j, n_{c, i_{s}^{c}}, A_{s}\right\rangle$ for $A_{s} \prec X$ is bounded by $2^{-c+1}$, and $L$ is an oracle KC set. Thus, by Lemma 2.8, for each $s>t$ we have

$$
K_{s+1}^{A_{s}}\left(n_{c, i_{s}^{c}}\right) \leqslant c-\log u_{c, s}+k=c+k-\log \sum_{\sigma \in C\left(m_{\left.c, l_{s}, s\right)}\right.} 2^{-K_{s}^{A_{s}}(\sigma)}
$$

which gives us the desired inequality.

Lemma 2.11. A has finite self-information.

Proof. We must show that the weight of $M$ is finite, and hence $M$ is actually a KC set. Let $t$ be as in Lemma 2.10 . Let $\widehat{M}$ be the portion of $M$ enumerated after stage $t$. It is enough to show that the weight of $\widehat{M}$ is finite.

We enumerate $\left\langle K_{s}^{A_{s}}(\sigma)+f_{s}(\sigma), \sigma\right\rangle$ into $\widehat{M}$ only when we have $K_{s}^{A_{s}}(\sigma)+f_{s}(\sigma)<K_{u}^{A_{u}}(\sigma)+f_{u}(\sigma)$ for all $u<s$, so the weight of $\widehat{M}$ is bounded by $2 \sum_{\sigma} 2^{-\min _{u>t}\left(K_{u}^{A_{t}}(\sigma)+f_{u}(\sigma)\right)}$. For each $\sigma$, either

$$
\min _{u>t} K_{u}^{A_{u}}(\sigma)+f_{u}(\sigma)=K^{A}(\sigma)+f(\sigma),
$$

or there is an $s>t$ such that

$$
K_{s}^{A_{s}}(\sigma)+f_{s}(\sigma)=\min _{u>t} K_{u}^{A_{u}}(\sigma)+f_{u}(\sigma)
$$

and there is a minimal length $A_{s}$-description of $\sigma$ whose use is violated by an enumeration into $A$ at stage $s$. The amount contributed to the weight of $\widehat{M}$ by all $\sigma$ for which the former case holds is bounded above by $2 \sum_{\sigma} 2^{-\left(K^{A}(\sigma)+f(\sigma)\right)}<2 \sum_{\sigma} 2^{-K^{A}(\sigma)}<2$, so it is enough to bound the sum of $2^{-\left(K_{s}^{A_{s}}(\sigma)+f_{s}(\sigma)\right)}$ over all $\sigma$ and $s>t$ such that some minimal length $A_{s}$-description of $\sigma$ is violated by an enumeration into $A$ at stage $s$. For each such $\sigma$ and $s$, the enumeration into $A$ at stage $s$ is done for the sake of some particular $c$. Say that $c$ is responsible for $2^{-\left(K_{s}^{A_{s}}(\sigma)+f_{s}(\sigma)\right)}$. It is enough to fix $c$ and show that the sum of all $2^{-\left(K_{s}^{A_{s}}(\sigma)+f_{s}(\sigma)\right)}$ for which $c$ is responsible is bounded by $2^{-c+O(1)}$.

Let $i_{s}$ be the value of the counter associated with $c$ at stage $s$. As above, let $C(x, s)$ be the set of all $\sigma$ such that there is a minimal length $A_{s}$-description of $\sigma$ at stage $s$ with use greater than $x$. The cost of putting $x$ into $A$ at stage $s$ for the sake of $c$ (in terms of the sum we are trying to bound) is

$$
\sum_{\sigma \in C(x, s)} 2^{-K_{s}^{A_{s}}(\sigma)-f_{s}(\sigma)}
$$

Let $k$ be as in Lemma 2.10. Then, at stages $s>t$ at which the $c$ strategy can act, we have

$$
2^{-K_{s+1}\left(n_{c, i s}\right)+c} \geqslant 2^{-K_{s+1}^{A_{s}}\left(n_{c, i_{s}}\right)} \geqslant 2^{-c-k} \sum_{\sigma \in C\left(m_{c, i, s}, s\right)} 2^{-K_{s}^{A_{s}}(\sigma)} .
$$


So each time we put a number in $\left(m_{c, i_{s}}, n_{c, i_{s}}\right)$ into $A$ at a stage $s>t$, we add to our sum at most

$$
\sum_{\sigma \in C\left(m_{c, i_{s}}, s\right)} 2^{-K_{s}^{A_{s}}(\sigma)-f_{s}(\sigma)} \leqslant \sum_{\sigma \in C\left(m_{c, i s}, s\right)} 2^{-K_{s}^{A_{s}}(\sigma)-4 c} \leqslant 2^{-4 c} 2^{-K_{s+1}\left(n_{c, i s}\right)+2 c+k}=2^{-K_{s+1}\left(n_{c, i_{s}}\right)-2 c+k} .
$$

By Lemma 2.7, for each $i$ and each value of $K_{s}\left(n_{c, i}\right)$, we put at most $2^{c+b}$-many numbers in $\left(m_{c, i}, n_{c, i}\right)$ into $A$. For each stage $s$ at which we do so, except possibly for the last one, $K_{s+1}\left(n_{c, i}\right)=K_{s}\left(n_{c, i}\right)$. Thus our total cost is bounded by

$$
\left(2^{c+b}+1\right) \sum_{i} \sum_{j \geqslant K\left(n_{c, i}\right)} 2^{-j-2 c+k} \leqslant 2^{c+b+1} \sum_{i} 2^{-K\left(n_{c, i}\right)-2 c+k+2}=2^{-c+b+k+3} \sum_{i} 2^{-K\left(n_{c, i}\right)}<2^{-c+b+k+3} .
$$

Therefore, the weight of $\widehat{M}$ is bounded by $2+\sum_{c} 2^{-c+b+k+3}$, which is finite.

This lemma completes the proof of the theorem.

\section{Relationships to Other Weakness Notions}

Recent work has explored the relationship between $K$-triviality and jump-traceability, a notion of approximability of the jump operator. We begin with some definitions.

\section{Definition 3.1.}

(i) Define the jump operator $J$ by $J^{A}(e)=\Phi_{e}^{A}(e)$, where $\Phi_{e}$ is the $e$ th partial computable function.

(ii) $A$ is jump-traceable if there are a computable function $h$ and a uniformly c.e. family $\left\{T_{e}\right\}$ of subsets of $\omega$ such that $\forall e\left|T_{e}\right| \leqslant h(e)$ and $J^{A}(e) \downarrow \Rightarrow J^{A}(e) \in T_{e}$. If $h$ is known we say $A$ is jump-traceable via $h$.

(iii) A function $h$ is an order if it is computable, non-decreasing, and unbounded.

(iv) A is strongly jump-traceable if it is jump-traceable via every order.

Figueira, Nies, and Stephan [7] constructed a promptly simple strongly jump-traceable set, separating that class from the computable sets. Cholak, Downey, and Greenberg [4] showed that the class of strongly jump-traceable c.e. sets is properly contained in the class of $K$-trivial c.e. sets. Recently, Diamondstone, Greenberg, and Turetsky [5] showed that every strongly jump-traceable set is computable in a c.e. strongly jump-traceable set, which implies that the result in [4] holds without the assumption of computable enumerability.

The proof in [4] shows that if a c.e. set $A$ is jump-traceable via an order $h \approx \sqrt{\log n}$ then it is $K$-trivial. Nies [18] showed that all $K$-trivials are jump-traceable, and in particular that there exists a constant $C$ such that if $A$ is low for $K$ with constant $b$, then $A$ is jump-traceable with trace obtained effectively in $b$ and with bound $C 2^{b} n \log n$. This result led to the question of whether between $\sqrt{\log n}$ and $n \log n$ there is a growth rate cutoff that separates $K$-trivials from non- $K$-trivials. Hölzl, Kräling, and Merkle [9] did find such a growth rate, but not a computable one. Their result implies that every $K$-trivial is jump-traceable via an order that is $O(\log n)$. Recently, Turetsky [22] built a $K$-trivial that is not jump-traceable via any order that is $o(\log n)$.

Cholak, Downey, and Greenberg [4] asked in particular whether the $K$-trivials are exactly those sets that are $h$-jump-traceable for all convergent order functions $h$, those functions for which $\sum_{n} \frac{1}{h(n)}<\infty$. Containment of the $K$-trivials in this class of jump-traceables is a consequence of the decanter method used to show that all $K$-trivials are superlow. (See [6] or [20] for details on this method.) Barmpalias, Downey, and Greenberg [2], however, showed that this containment is proper. Hence $K$-triviality sits strictly between "jump-traceable for all order functions $h$ " (i.e., strongly jump-traceable) and "jump-traceable for all convergent order functions $h$ " (in fact "jump-traceable for all superlinear order functions", where superlinear means $\lim _{n} \frac{h(n)}{n}=\infty$ ).

We may ask where the sets that have finite self-information fall in this hierarchy. The following result and its proof are a first step in addressing this question. 
Proposition 3.2. If a set has finite self-information then it is jump-traceable.

Proof. Suppose $A$ is not jump-traceable. Let $\sigma_{0}, \sigma_{1}, \ldots$ be an effective listing of $2^{<\omega}$. If $J^{A}(e) \downarrow$ then let $\tau_{e}=\sigma_{J^{A}}(e)$. Let $L$ be the set of all pairs $\left\langle K_{s}(e), \tau_{e}\right\rangle$ such that $s \in \omega$ and $\tau_{e}$ is defined. It is easy to check that $L$ is a KC set relative to $A$, so if $\tau_{e}$ is defined then $K^{A}\left(\tau_{e}\right) \leqslant{ }^{+} K(e)$.

Let $T_{e}=\left\{i \mid K\left(\sigma_{i}\right)<4 \log e\right\}$. The $T_{e}$ are uniformly c.e. and $\left|T_{e}\right|<e^{4}$. Since $A$ is not jump-traceable, there must be infinitely many $e$ such that $J^{A}(e) \notin T_{e}$. For any such $e$, we have $K\left(\tau_{e}\right) \geqslant 4 \log e$. Let $S$ be the set of such $e$. Since $K(e) \leqslant 2 \log e+O(1)$, we have

$$
I(A: A)>\log \sum_{e \in S} 2^{K\left(\tau_{e}\right)-2 K^{A}\left(\tau_{e}\right)-O(1)} \geqslant \log \sum_{e \in S} 2^{4 \log e-2 K(e)-O(1)}=\infty .
$$

From the proof we see that if a set has finite self-information then it is jump-traceable via the order $n^{4}$. Since $K(e) \leqslant \log e+2 \log \log e+O(1)$, we may replace the $4 \log e$ in the definition of $T_{e}$ by $2 \log e+4 \log \log e$, so in fact, if a set has finite self-information then it is jump-traceable via any $n^{2+\epsilon}$ for $\epsilon>0$. We do not know whether every set that has finite self-information is jump-traceable via $n^{2}$, say, so our results leave open the exact relationships between having finite self-information and various interesting subclasses of the jump-traceables, such as the class of sets that are jump-traceable for all convergent order functions.

Recall that a set $A$ is generalized low, or $G L_{1}$, if $A^{\prime} \leqslant_{\mathrm{T}} A \oplus \emptyset^{\prime}$. It follows from the above result that if a set $A$ has finite self-information, then it is $G L_{1}$ (see Proposition 8.4.3 of [20]). If $A$ is also $\Delta_{2}^{0}$, it is therefore low, in the usual computability theoretic sense. However, Herbert [8] has recently shown that there is a perfect $\Pi_{1}^{0}$ class of sets that have finite self-information, which in particular answers a question in a previous version of this paper by showing the existence of non- $\Delta_{2}^{0}$ sets with finite self-information.

Lutz [16] and others have developed an effective version of Hausdorff dimension based on martingales in inflationary environments, called $s$-gales. From success of $s$-gales we have the constructive dimension, or effective Hausdorff dimension, $\operatorname{dim}(S)$, and from strong success of $s$-gales we have constructive strong dimension, or effective packing dimension, $\operatorname{Dim}(S)$. (See Chapter 13 of [6] for more details.) These dimensions have a deep tie to randomness, as shown by the following results.

Theorem 3.3. Let $S \in 2^{\omega}$.

(i) (Mayordomo [17]). $\operatorname{dim}(S)=\liminf _{n \rightarrow \infty} \frac{K(S\lceil n)}{n}$.

(ii) (Athreya, Hitchcock, Lutz, and Mayordomo [1]).

$$
\operatorname{Dim}(S)=\limsup _{n \rightarrow \infty} \frac{K(S\lceil n)}{n} .
$$

Effective dimension and the theorem above may be relativized, giving rise to the notion of lowness for dimension. A set $A$ is low for effective Hausdorf dimension if the effective Hausdorff dimension of $S$ relative to $A$ is equal to the effective Hausdorff dimension of $S$ for all $S$. We can define lowness for effective packing dimension similarly. Clearly, all $K$-trivial sequences are low for both notions of dimension. In the proof of Theorem 2.2, if instead of ensuring $K(\sigma) \leqslant{ }^{+} K^{A}(\sigma)+f(\sigma)$ for the $f$ of Lemma 2.3 we instead ensure it for $f(\sigma)=\log |\sigma|$, then for all $S$,

$$
\frac{K(S\lceil n)}{n} \leqslant \frac{K^{A}(S\lceil n)}{n}+\frac{\log n}{n},
$$

and the last fraction has limsup and lim inf zero. Hence, a set $A$ built in this way will be low for both effective Hausdorff and effective packing dimension, but not $K$-trivial, yielding the following result.

Theorem 3.4. There is a c.e. set that is low for both effective Hausdorff dimension and effective packing dimension but is not $K$-trivial. 
In the forthcoming paper [11], Lempp, Miller, Ng, Turetsky, and Weber show that there is in fact a perfect $\Pi_{1}^{0}$ class of sets that are low for effective Hausdorff dimension, a result that also follows by the methods of Herbert [8].

The function $f$ from Lemma 2.3 grows much more slowly than $\log |\sigma|$, so it seems to be much easier to produce a set that is low for dimension than one that has finite self-information. On the other hand, Lempp, Miller, $\mathrm{Ng}$, Turetsky, and Weber [11] show that if a set is low for effective Hausdorff dimension then it is jump traceable via $O\left(h^{r}\right)$ for any convergent order function $h$ and any $r>1$, and thus, in particular, via $n^{1+\epsilon}$ for any $\epsilon>0$, which is a better bound than what we obtained above for sets that have finite self-information.

Question 3.5. What is the exact relationship between having finite self-information and lowness for dimension?

\section{References}

[1] K. B. Athreya, J. M. Hitchcock, J. H. Lutz, and E. Mayordomo. Effective strong dimension in algorithmic information and computational complexity. SIAM Journal on Computing, 37:671-705, 2007.

[2] G. Barmpalias, R. G. Downey, and N. Greenberg. K-trivial degrees and the jump-traceability hierarchy. Proceedings of the American Mathematical Society, 137:2099-2109, 2009.

[3] G. J. Chaitin. Information-theoretical characterizations of recursive infinite strings. Theoretical Computer Science, 2:45-48, 1976.

[4] P. A. Cholak, R. G. Downey, and N. Greenberg. Strong jump-traceability I: The computably enumerable case. Advances in Mathematics, 217:2054-2074, 2008.

[5] D. Diamondstone, N. Greenberg, and D. Turetsky. Inherent enumerability of strong jump-traceability, To appear.

[6] R. G. Downey, and D. R. Hirschfeldt. Algorithmic Randomness and Complexity. Theory and Applications of Computability, Springer, New York, 2010.

[7] S. Figueira, A. Nies, and F. Stephan. Lowness properties and approximations of the jump. Annals of Pure and Applied Logic, 152:51-66, 2008.

[8] I. Herbert. A Perfect set of Reals Low for Information. Preprint.

[9] R. Hölzl, T. Kräling, and W. Merkle. Time bounded Kolmogorov complexity and Solovay functions. In R. Královič and D. Niwiński, editors, Mathematical Foundations of Computer Science 2009. Lecture Notes in Computer Science 5734, pages 392-402. Springer, Berlin, 2009.

[10] A. N. Kolmogorov. Three approaches for defining the concept of information quantity. Problems of Information Transmission, 1:1-7, 1965.

[11] S. Lempp, J. S. Miller, K. M. Ng, D. Turetsky, and R. Weber. Lowness for effective Hausdorff dimension, To appear.

[12] L. A. Levin. Laws of information conservation (nongrowth) and aspects of the foundation of probability theory. Problems of Information Transmission, 10:206-210, 1974.

[13] L. A. Levin. Randomness conservation inequalities; information and independence of mathematical theories. Information and Control, 61:15-37, 1984.

[14] L. A. Levin. Forbidden Information. Preprint (arXiv:cs/0203029v16).

[15] M. Li and P. Vitányi. An Introduction to Kolmogorov Complexity and its Applications. Texts in Computer Science. Springer, New York, 3rd edition, 2008.

[16] J. H. Lutz. The dimensions of individual strings and sequences. Information and Computation, 187:49-79, 2003. 
[17] E. A. Mayordomo. Kolmogorov complexity characterization of constructive Hausdorff dimension. Information Processing Letters, 84:1-3, 2002.

[18] A. Nies. Reals which compute little. In Z. Chatzidakis, P. Koepke, and W. Pohlers, editors, Proceedings of Logic Colloquium 2002. Lecture Notes in Logic, Vol. 27, pages 261-275. 2002.

[19] A. Nies. Lowness properties and randomness. Advances in Mathematics, 197:274-305, 2005.

[20] A. Nies. Computability and Randomness. Oxford Logic Guides 51. Oxford University Press, Oxford, 2009.

[21] R. I. Soare. Recursively Enumerable Sets and Degrees. Perspectives in Mathematical Logic. Springer-Verlag, Berlin, 1987.

[22] D. Turetsky. $K$-trivial sets and jump traceability, To appear. 